\title{
Investigation of Numerical Stability of Electromagnetic Wave Propagation Simulation using Meshless Time-Domain Method ${ }^{*)}$
}

\author{
Taku ITOH, Yoshihisa FUJITA, Soichiro IKUNO and Hiroaki NAKAMURA ${ }^{1)}$ \\ Tokyo University of Technology, 1404-1 Katakura-machi, Hachioji, Tokyo 192-0982, Japan \\ 1) National Institute for Fusion Science, 322-6 Oroshi-cho, Toki, Gifu 509-5292, Japan
}

(Received 10 December 2012 / Accepted 13 April 2013)

\begin{abstract}
To investigate a condition of the numerical stability of an electromagnetic wave propagation simulation using the meshless time-domain method (MTDM), a 1-dimensional (1D) TM mode discretized by the MTDM has been analyzed theoretically. Under some assumptions, the Courant condition for the 1D MTDM has been derived. The Courant condition does not depend on the radial basis functions required to generate shape functions of the MTDM. In addition, the Courant condition for the 1D MTDM is equivalent to that for the finite-difference timedomain method. Based on the result for the 1D case, the Courant condition for the 2-dimensional (2D) MTDM is predicted. Furthermore, for the case where the predicted Courant condition is satisfied, the numerical stability of 2D MTDM has been investigated numerically.
\end{abstract}

(C) 2013 The Japan Society of Plasma Science and Nuclear Fusion Research

Keywords: meshless time-domain method, finite-difference time-domain method, shape functions of meshless methods, courant condition, electromagnetic wave propagation simulation

DOI: $10.1585 /$ pfr.8.2401101

\section{Introduction}

The finite-difference time-domain method (FDTD) has generally been applied for electromagnetic wave propagation simulations, and it has yielded many impressive results. In numerical simulations employing the FDTD, the numerical domain is divided into rectangular meshes. However, it is difficult to accurately represent an arbitraryshaped domain using only rectangular meshes.

On the other hand, many kinds of meshless methods such as the element-free Galerkin method [1], the meshless local Petrov-Galerkin method [2] and the meshless radial point interpolation method (RPIM) [3] have been proposed, and applied to numerical simulations in various fields, including plasma physics and fusion science. In the meshless methods, the shape functions are generated from nodes, i.e., the meshes are no longer necessary.

Recently, the meshless method based on the RPIM has been applied to electromagnetic wave propagation simulations [4]. This method is called here the meshless timedomain method (MTDM). In the MTDM, a domain is discretized by the shape functions of the RPIM. Namely, the MTDM does not require the rectangular meshes, which are required in the FDTD. Hence, the node alignment of the MTDM is more flexible than that of the FDTD. However, in the MTDM, the relation between the numerical stability and the node alignment has not been investigated sufficiently.

The purpose of the present study is to investigate a

author'se-mail: taku@m.ieice.org

*) This article is based on the presentation at the 22nd International Toki Conference (ITC22). condition of the numerical stability of the MTDM in electromagnetic wave propagation simulations. To this end, a 1-dimensional (1D) Transverse Magnetic (TM) mode discretized by the MTDM is analyzed theoretically.

\section{Shape Functions of the RPIM}

In the MTDM, the leap-frog method is employed to discretize the time domain, and the space domain is discretized by the shape functions of the RPIM [4]. The shape functions are derived by the following procedures.

First, the nodes, $\boldsymbol{x}_{1}, \boldsymbol{x}_{2}, \ldots, \boldsymbol{x}_{N}$, together with the radial basis functions (RBFs), $w_{1}(\boldsymbol{x}), w_{2}(\boldsymbol{x}), \ldots, w_{N}(\boldsymbol{x})$, on each of the nodes are assigned in the domain $\Omega$ and on the boundary $\partial \Omega$, where $N$ is the number of nodes, and $w_{i}(\boldsymbol{x}) \equiv w\left(\left|\boldsymbol{x}-\boldsymbol{x}_{i}\right|\right)(i=1,2, \ldots, N)$. In the RPIM, it is assumed that the solution $u(\boldsymbol{x})$ can be expanded by

$$
u(\boldsymbol{x})=\left[\boldsymbol{w}^{\mathrm{T}}(\boldsymbol{x}), \boldsymbol{p}^{\mathrm{T}}(\boldsymbol{x})\right] G^{-1}\left[\begin{array}{c}
\boldsymbol{u}^{\mathrm{e}} \\
\mathbf{0}
\end{array}\right]=\boldsymbol{\phi}^{\mathrm{T}}(\boldsymbol{x}) \boldsymbol{u}^{\mathrm{e}} .
$$

Here, the vectors, $\boldsymbol{w}(\boldsymbol{x}), \boldsymbol{p}(\boldsymbol{x}), \boldsymbol{u}^{\mathrm{e}}$ and $\boldsymbol{\phi}(\boldsymbol{x})$ are, respectively, defined by

$$
\begin{aligned}
\boldsymbol{w}(\boldsymbol{x}) & \equiv\left[w_{1}(\boldsymbol{x}), w_{2}(\boldsymbol{x}), \ldots, w_{N}(\boldsymbol{x})\right]^{\mathrm{T}}, \\
\boldsymbol{p}(\boldsymbol{x}) & \equiv\left[p_{1}(\boldsymbol{x}), p_{2}(\boldsymbol{x}), \ldots, p_{M}(\boldsymbol{x})\right]^{\mathrm{T}}, \\
\boldsymbol{u}^{\mathrm{e}} & \equiv\left[u_{1}^{\mathrm{e}}, u_{2}^{\mathrm{e}}, \ldots, u_{N}^{\mathrm{e}}\right]^{\mathrm{T}}, \\
\boldsymbol{\phi}(\boldsymbol{x}) & \equiv\left[\phi_{1}(\boldsymbol{x}), \phi_{2}(\boldsymbol{x}), \ldots, \phi_{N}(\boldsymbol{x})\right]^{\mathrm{T}},
\end{aligned}
$$

where $\phi_{k}(\boldsymbol{x})$ denotes a shape function corresponding to the $k$ th node $\boldsymbol{x}_{k}(k=1,2, \ldots, N)$, and $M$ is the number of components of $\boldsymbol{p}(\boldsymbol{x})$. In this study, for the 2-dimensional (2D) case, $M=3$ is adopted, i.e., $\boldsymbol{p}(\boldsymbol{x})=[1, x, y]^{\mathrm{T}}$, whose com- 
ponents are coefficients of a degree-one polynomial. In addition, the matrix $G \in \mathbb{R}^{(N+M) \times(N+M)}$ is defined by

$$
G \equiv\left[\begin{array}{cc}
W & P \\
P^{\mathrm{T}} & O
\end{array}\right]
$$

where $W \equiv\left[\boldsymbol{w}\left(\boldsymbol{x}_{1}\right), \boldsymbol{w}\left(\boldsymbol{x}_{2}\right), \ldots, \boldsymbol{w}\left(\boldsymbol{x}_{N}\right)\right]^{\mathrm{T}}$ and $P \equiv\left[\boldsymbol{p}\left(\boldsymbol{x}_{1}\right)\right.$, $\left.\boldsymbol{p}\left(\boldsymbol{x}_{2}\right), \ldots, \boldsymbol{p}\left(\boldsymbol{x}_{N}\right)\right]^{\mathrm{T}}$. From (1), the explicit form of the shape functions $\phi_{k}(\boldsymbol{x})(k=1,2, \ldots, N)$ and these derivatives can be expressed by

$$
\begin{gathered}
\phi_{k}(\boldsymbol{x})=\sum_{i=1}^{N} w_{i}(\boldsymbol{x}) \bar{g}_{i, k}+\sum_{j=1}^{M} p_{j}(\boldsymbol{x}) \bar{g}_{N+j, k}, \\
\frac{\partial \phi_{k}}{\partial x}(\boldsymbol{x})=\sum_{i=1}^{N} \frac{\partial w_{i}}{\partial x}(\boldsymbol{x}) \bar{g}_{i, k}+\sum_{j=1}^{M} \frac{\partial p_{j}}{\partial x}(\boldsymbol{x}) \bar{g}_{N+j, k}, \\
\frac{\partial \phi_{k}}{\partial y}(\boldsymbol{x})=\sum_{i=1}^{N} \frac{\partial w_{i}}{\partial y}(\boldsymbol{x}) \bar{g}_{i, k}+\sum_{j=1}^{M} \frac{\partial p_{j}}{\partial y}(\boldsymbol{x}) \bar{g}_{N+j, k},
\end{gathered}
$$

where $\bar{g}_{i, k}$ denotes the $(i, k)$-element of the matrix $G^{-1}$. It must be noted here that the shape functions have the Kronecker delta function property as follows [3]:

$$
\phi_{i}\left(\boldsymbol{x}_{j}\right)= \begin{cases}1 & \text { for } i=j \\ 0 & \text { for } i \neq j\end{cases}
$$

\section{Meshless Time-Domain Method}

For simplicity, we consider 2D electromagnetic wave propagation of the TM mode whose governing equations are described by

$$
\begin{aligned}
\varepsilon \frac{\partial E_{z}}{\partial t} & =-\sigma E_{z}+\frac{\partial H_{y}}{\partial x}-\frac{\partial H_{x}}{\partial y}, \\
\mu \frac{\partial H_{x}}{\partial t} & =-\frac{\partial E_{z}}{\partial y}, \\
\mu \frac{\partial H_{y}}{\partial t} & =\frac{\partial E_{z}}{\partial x},
\end{aligned}
$$

where $E_{z}$ denotes the $z$ component of the electric field, and $H_{x}$ and $H_{y}$ denote the $x$ and $y$ components of the magnetic field, respectively. In addition, $\varepsilon, \sigma$, and $\mu$ denote the permittivity, electrical conductivity, and magnetic permeability, respectively.

To discretize (11), (12), and (13), nodes $\boldsymbol{x}_{i}^{E}(i=$ $\left.1,2, \ldots, N_{E}\right)$ for $E_{z}$ and $\boldsymbol{x}_{i}^{H}\left(i=1,2, \ldots, N_{H}\right)$ for $H_{x}$ and $H_{y}$ are first aligned in a domain, where $N_{E}$ denotes the number of nodes for $E_{z}$, and $N_{H}$ denotes the number of nodes for $H_{x}$ and $H_{y}$. As mentioned in Section 2, in the MTDM, the leap-frog method is employed to discretize the time domain. In addition, the space domain is discretized by the shape functions of the RPIM. The discretized forms of (11), (12) and (13) are as follows:

$$
E_{z, i}^{n}=\frac{\left(\frac{\varepsilon}{\Delta t}-\frac{\sigma}{2}\right) E_{z, i}^{n-1}+\sum_{j=1}^{N_{H}} H_{y, j}^{n-\frac{1}{2}} \frac{\partial \phi_{j, i}^{H}}{\partial x}-\sum_{j=1}^{N_{H}} H_{x, j}^{n-\frac{1}{2}} \frac{\partial \phi_{j, i}^{H}}{\partial y}}{\left(\frac{\varepsilon}{\Delta t}+\frac{\sigma}{2}\right)},
$$

$$
\begin{aligned}
& H_{x, i}^{n+\frac{1}{2}}=H_{x, i}^{n-\frac{1}{2}}-\frac{\Delta t}{\mu} \sum_{j=1}^{N_{E}} E_{z, j}^{n} \frac{\partial \phi_{j, i}^{E}}{\partial y} \\
& H_{y, i}^{n+\frac{1}{2}}=H_{y, i}^{n-\frac{1}{2}}+\frac{\Delta t}{\mu} \sum_{j=1}^{N_{E}} E_{z, j}^{n} \frac{\partial \phi_{j, i}^{E}}{\partial x}
\end{aligned}
$$

where $n$ is the time step, $E_{z, i}^{n} \equiv E_{z}^{n}\left(\boldsymbol{x}_{i}^{E}\right), H_{x, i}^{n+\frac{1}{2}} \equiv H_{x}^{n+\frac{1}{2}}\left(\boldsymbol{x}_{i}^{H}\right)$,

$$
\begin{aligned}
& \frac{\partial \phi_{j, i}^{E}}{\partial x} \equiv \frac{\partial \phi_{j}^{E}}{\partial x}\left(\boldsymbol{x}_{i}^{H}\right), \quad \frac{\partial \phi_{j, i}^{E}}{\partial y} \equiv \frac{\partial \phi_{j}^{E}}{\partial y}\left(\boldsymbol{x}_{i}^{H}\right), \\
& \frac{\partial \phi_{j, i}^{H}}{\partial x} \equiv \frac{\partial \phi_{j}^{H}}{\partial x}\left(\boldsymbol{x}_{i}^{E}\right), \text { and } \frac{\partial \phi_{j, i}^{H}}{\partial y} \equiv \frac{\partial \phi_{j}^{H}}{\partial y}\left(\boldsymbol{x}_{i}^{E}\right) .
\end{aligned}
$$

In addition, $\phi_{j}^{E}(\boldsymbol{x})$ denotes the shape functions corresponding to $\boldsymbol{x}_{j}^{E}\left(j=1,2, \ldots, N_{E}\right)$. Similarly, $\phi_{j}^{H}(\boldsymbol{x})$ denotes the shape functions corresponding to $\boldsymbol{x}_{j}^{H}\left(j=1,2, \ldots, N_{H}\right)$. Note that, to derive (14), (15), and (16) from (11), (12), and (13), respectively, the Kronecker delta function property (10) is used, e.g., $E_{z}^{n-1}(\boldsymbol{x})$ is expanded as

$$
E_{z}^{n-1}(\boldsymbol{x})=\sum_{j=1}^{N_{E}} E_{z, j}^{n-1} \phi_{j}^{E}(\boldsymbol{x})
$$

and by substituting $\boldsymbol{x}=\boldsymbol{x}_{i}^{E}$,

$$
E_{z}^{n-1}\left(\boldsymbol{x}_{i}^{E}\right)=\sum_{j=1}^{N_{E}} E_{z, j}^{n-1} \phi_{j}^{E}\left(\boldsymbol{x}_{i}^{E}\right)=E_{z, i}^{n-1}
$$

In addition, an approximation $E_{z} \simeq\left(E_{z}^{n}+E_{z}^{n-1}\right) / 2$ is adopted to derive (14) from (11). By calculating (14), (15), and (16) in each time step, the behavior of the electromagnetic wave propagation can be simulated.

\section{Condition for Numerical Stability}

In this section, to analyze a condition of the numerical stability of the MTDM, we consider a 1D TM mode as follows:

$$
\begin{aligned}
\varepsilon \frac{\partial E_{z}}{\partial t} & =\frac{\partial H_{y}}{\partial x}, \\
\mu \frac{\partial H_{y}}{\partial t} & =\frac{\partial E_{z}}{\partial x} .
\end{aligned}
$$

To discretize (21) and (22), nodes $x_{i}\left(i=1,2, \ldots, N_{E}\right)$ for $E_{z}$ and $x_{i+\frac{1}{2}}\left(i=1,2, \ldots, N_{H}\right)$ for $H_{y}$ are first aligned. Here, we assume that the nodes are uniformly aligned so that $\Delta x=\left|x_{i}-x_{i+1}\right|$ is the same value. In addition, throughout this section, we assume $x_{i+\frac{1}{2}}=x+\Delta x / 2$ and $N_{H}=N_{E}-1$.

With the above assumptions, by utilizing the leap-frog method and the shape functions of the RPIM, (21) and (22) are discretized as

$$
\begin{gathered}
E_{z, i}^{n}=E_{z, i}^{n-1}+\frac{\Delta t}{\varepsilon} \sum_{j=1}^{N_{H}} H_{y, j+\frac{1}{2}}^{n-\frac{1}{2}} \frac{\partial \phi_{j, i}^{H}}{\partial x}, \\
H_{y, i+\frac{1}{2}}^{n+\frac{1}{2}}=H_{y, i+\frac{1}{2}}^{n-\frac{1}{2}}+\frac{\Delta t}{\mu} \sum_{j=1}^{N_{E}} E_{z, j}^{n} \frac{\partial \phi_{j, i}^{E}}{\partial x} .
\end{gathered}
$$




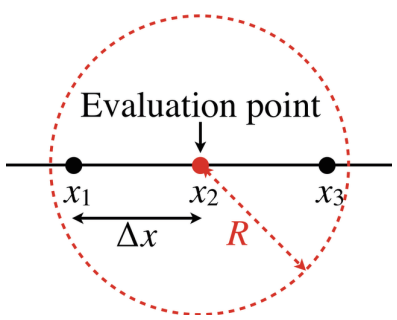

Fig. 1 Schematic view of node alignment, the evaluation point $x_{2}$, and the support radius $R$ of an RBF for theoretically analyzing a condition of the numerical stability. Here, $\Delta x=\left|x_{2}-x_{1}\right|=\left|x_{3}-x_{2}\right|$.

To theoretically analyze a condition of the numerical stability, we determine the explicit form of the derivatives of shape functions. To this end, in the following, we assume that the number of evaluation points inside the support radius is 3 , and $x_{2}$ is the evaluation point (see Fig. 1). Under these assumptions, $\frac{\partial \phi}{\partial x}\left(x_{2}\right)$ can be determined by solving the following linear system.

$$
\left[\begin{array}{ccccc}
w_{1}\left(x_{1}\right) & w_{2}\left(x_{1}\right) & w_{3}\left(x_{1}\right) & 1 & x_{1} \\
w_{1}\left(x_{2}\right) & w_{2}\left(x_{2}\right) & w_{3}\left(x_{2}\right) & 1 & x_{2} \\
w_{1}\left(x_{3}\right) & w_{2}\left(x_{3}\right) & w_{3}\left(x_{3}\right) & 1 & x_{3} \\
1 & 1 & 1 & 0 & 0 \\
x_{1} & x_{2} & x_{3} & 0 & 0
\end{array}\right] \frac{\partial \phi}{\partial x}=\left[\begin{array}{c}
\frac{\partial w_{1}}{\partial x} \\
0 \\
\frac{\partial w_{3}}{\partial x} \\
0 \\
1
\end{array}\right] .
$$

From (25),

$$
\frac{\partial \boldsymbol{\phi}}{\partial x}\left(x_{2}\right)=\left[-\frac{1}{2 \Delta x}, 0, \frac{1}{2 \Delta x}, c_{1}, c_{2}\right]^{\mathrm{T}},
$$

where $c_{1}$ and $c_{2}$ are constant values that are not used in the MTDM. It must be noted here that, regardless of the RBFs, (26) becomes the same result except for $c_{1}$ and $c_{2}$. By substituting (26) into (23) and (24), these equations are rewritten as

$$
\begin{aligned}
E_{z, i}^{n} & =E_{z, i}^{n-1}+\frac{\Delta t}{\varepsilon \Delta x}\left(H_{y, i+\frac{1}{2}}^{n-\frac{1}{2}}-H_{y, i-\frac{1}{2}}^{n-\frac{1}{2}}\right), \\
H_{y, i+\frac{1}{2}}^{n+\frac{1}{2}} & =H_{y, i+\frac{1}{2}}^{n-\frac{1}{2}}+\frac{\Delta t}{\mu \Delta x}\left(E_{z, i+1}^{n}-E_{z, i}^{n}\right) .
\end{aligned}
$$

Using (27) and (28), we investigate the Courant condition for the MTDM. To this end, we assume that the input wave is a plane wave described by

$$
\begin{aligned}
& \boldsymbol{E}(\boldsymbol{r}, t)=\boldsymbol{E}_{0}(t) \mathrm{e}^{-j \boldsymbol{k} \cdot \boldsymbol{r}}, \\
& \boldsymbol{H}(\boldsymbol{r}, t)=\boldsymbol{H}_{0}(t) \mathrm{e}^{-j \boldsymbol{k} \cdot \boldsymbol{r}},
\end{aligned}
$$

where $j$ is the imaginary unit, $\boldsymbol{k}$ is the wave vector, and $\boldsymbol{r}$ is the space vector. Here, $\boldsymbol{k} \cdot \boldsymbol{r}=k_{x} x+k_{y} y+k_{z} z$. After (27) is substituted into (28), by substituting (29) and (30) into (27) and (28), these equations are described as

$$
\begin{aligned}
E_{z, 0}^{n} & =E_{z, 0}^{n-1}+j \frac{\Delta t S}{\varepsilon} H_{y, 0}^{n-\frac{1}{2}}, \\
H_{y, 0}^{n+\frac{1}{2}} & =H_{y, 0}^{n-\frac{1}{2}}-(v \Delta t S)^{2} H_{y, 0}^{n-\frac{1}{2}}+j \frac{\Delta t S}{\mu} E_{z, 0}^{n-1},
\end{aligned}
$$

where $v^{2}=(\mu \varepsilon)^{-1}$ and

$$
S=\frac{2 \sin \left(k_{x} \frac{\Delta x}{2}\right)}{\Delta x} .
$$

Equations (31) and (32) can be rewritten as

$$
\boldsymbol{x}^{n}=A \boldsymbol{x}^{n-1},
$$

where

$$
\boldsymbol{x}^{n} \equiv\left[\begin{array}{c}
E_{z, 0}^{n} \\
H_{y, 0}^{n+\frac{1}{2}}
\end{array}\right], \quad A \equiv\left[\begin{array}{cc}
1 & j \frac{\Delta t S}{\varepsilon} \\
j \frac{\Delta t S}{\mu} & 1-(v \Delta t S)^{2}
\end{array}\right] .
$$

In (34), if all eigenvalues $\lambda$ of $A$ satisfy $|\lambda| \leq 1$, the vector $\boldsymbol{x}^{n}$ will be converged to a stability solution when $n \rightarrow \infty$. These eigenvalues are determined by solving $|A-\lambda I|=0$, i.e.,

$$
\lambda=\frac{-b \pm \sqrt{b^{2}-4}}{2}
$$

where $b \equiv(v \Delta t S)^{2}-2$. In (36), if $b^{2}-4 \leq 0$, that is,

$$
(v \Delta t S)^{2} \leq 4,
$$

is satisfied, all eigenvalues of $A$ satisfy $|\lambda| \leq 1$. Furthermore, in (33), since $\left|\sin \left(k_{x} \frac{\Delta x}{2}\right)\right| \leq 1$ is satisfied,

$$
|S| \leq \frac{2}{\Delta x} .
$$

From (37) and (38), the Courant condition for the 1D MTDM is determined as

$$
v \Delta t \leq \Delta x
$$

From (39), we see that the Courant condition for the 1D MTDM does not depend on RBFs. In addition, it must be noted here that, under the above assumptions, the Courant condition for the 1D MTDM is equivalent to that for the FDTD. Furthermore, from (39), we consider that the Courant condition for the MTDM for 2D/3D depends on the adjacent node distance. Namely, we predict the Courant condition for the MTDM for 2D/3D as

$$
v \Delta t \leq \min \left|\boldsymbol{x}_{i}-\boldsymbol{x}_{j}\right|
$$

In the next section, for the case where (40) is satisfied, we investigate the influence of the adjacent node distance to the numerical stability for the $2 \mathrm{D}$ case.

\section{Numerical Experiments}

In this section, for the case where (40) is satisfied, numerical experiments were conducted to investigate the influence of the adjacent node distance to the numerical stability of a 2D electromagnetic wave propagation simulation using the MTDM. To this end, the line-shaped waveguide illustrated in Fig. 2 (a) is used for this simulation. In 


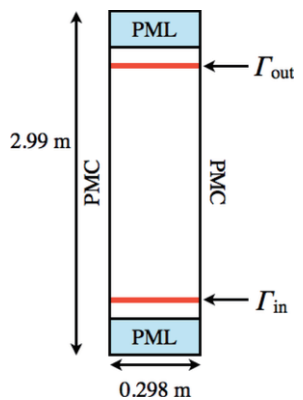

(a)

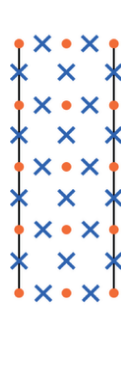

(b)

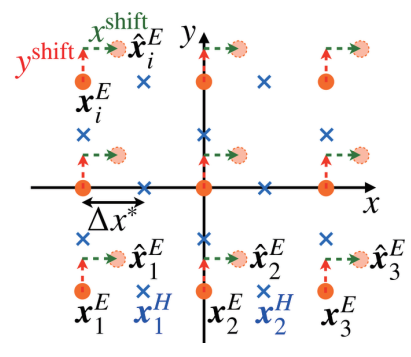

(c)

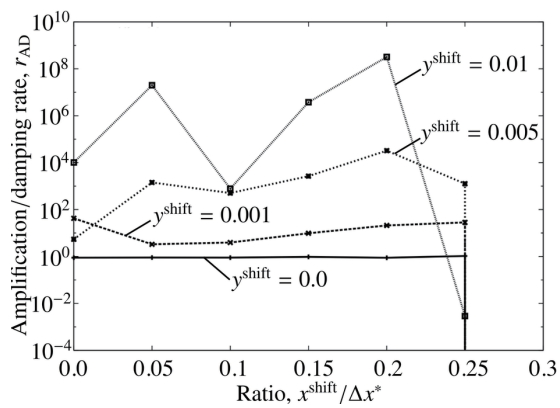

(d)

Fig. 2 (a) Schematic view of a line-shaped wave guide. Here, PML and PMC denote the perfectly matched layer and perfect magnetic conductor, respectively. In addition, $\Gamma_{\mathrm{in}}$ and $\Gamma_{\text {out }}$ denote the source input line and the observation line, respectively. (b) Schematic view of node alignment of $\boldsymbol{x}^{E}$ and that of $\boldsymbol{x}^{H}$. Here, $\boldsymbol{x}^{E}$ and $\boldsymbol{x}^{H}$ are represented as $\bullet$ and $\times$, respectively. (c) Schematic view of moving nodes $\boldsymbol{x}_{i}^{E}$. (d) Dependence of the amplification/damping rate $r_{\mathrm{AD}}$ on the ratio $x^{\text {shift }} / \Delta x^{*}$.

addition, the nodes $\boldsymbol{x}_{i}^{E}$ and $\boldsymbol{x}_{i}^{H}$ are uniformly aligned in alternate shifts as shown in Fig. 2 (b). In this simulation, we assume that the wave source is a sine wave whose amplitude, frequency and speed are $1.0(\mathrm{~V} / \mathrm{m}), 1.0 \times 10^{9}(\mathrm{~Hz})$ and $299792458(\mathrm{~m} / \mathrm{s})$, respectively. It must be noted here that, throughout this section, we set $\Delta t=0.8 \min \left|\boldsymbol{x}_{i}-\boldsymbol{x}_{j}\right| / v$ to satisfy (40).

As an RBF, we adopt the reciprocal multi quadric (RMQ):

$$
w_{i}(\boldsymbol{x})= \begin{cases}{\left[\left(\frac{\left|\boldsymbol{x}-\boldsymbol{x}_{i}\right|}{R_{i}}\right)^{2}+1\right]^{-\frac{1}{2}}} & \text { for }\left|\boldsymbol{x}-\boldsymbol{x}_{i}\right| \leq R_{i}, \\ 0 & \text { for }\left|\boldsymbol{x}-\boldsymbol{x}_{i}\right|>R_{i},\end{cases}
$$

where $R_{i}$ denotes the support radius of $w_{i}(\boldsymbol{x})$. Each support radius $R_{i}$ of $w_{i}(\boldsymbol{x})$ is determined so that the number of nodes inside the support radius is at least 12 .

To investigate the relation between the numerical stability and the adjacent node distance, we simultaneously move the nodes $\boldsymbol{x}_{i}^{E}=\left[x_{i}^{E}, y_{i}^{E}\right]^{\mathrm{T}}$ in the $x$-direction after moving $\boldsymbol{x}_{i}^{E}$ to $\boldsymbol{x}_{i}^{s}=\left[x_{i}^{E}, y_{i}^{E}+y^{\text {shift }}\right]^{\mathrm{T}}\left(i=1,2, \ldots, N_{E}\right)$. For each $\boldsymbol{x}_{i}^{E}$, four types of $\boldsymbol{x}_{i}^{s}$ are adopted by setting $y^{\text {shift }}=0.0,0.001,0.005$, and 0.01 . Here, we assume that $\boldsymbol{x}_{i}^{E}$ is moved to $\hat{\boldsymbol{x}}_{i}^{E}=\left[x_{i}^{E}+x^{\text {shift }}, y_{i}^{E}+y^{\text {shift }}\right]^{\mathrm{T}}$ as shown in Fig. 2 (c). For each $y^{\text {shift }}$, the dependence of the amplification/damping rate $r_{\mathrm{AD}}$ in the line-shaped waveguide on a ratio $x^{\text {shift }} / \Delta x^{*}$ is shown in Fig. $2(\mathrm{~d})$, where $\Delta x^{*}=\Delta x / 2$ and

$$
r_{\mathrm{AD}} \equiv\left\langle\int_{\Gamma_{\text {out }}} E_{z} \mathrm{~d} \ell\right\rangle_{t} /\left\langle\int_{\Gamma_{\text {in }}} E_{z} \mathrm{~d} \ell\right\rangle_{t} .
$$

Here, $\Gamma_{\text {in }}$ and $\Gamma_{\text {out }}$ denote the source input line and an observation line, respectively (see Fig. 2 (a)). In addition, $r_{\mathrm{AD}}$ has been calculated on $E_{z}$ past the line $\Gamma_{\text {out }}$ in a certain time step. We see from Fig. 2(d) that, for $y^{\text {shift }}=0.0$, $r_{\mathrm{AD}}=1.0$ can almost be maintained in $x^{\text {shift }} / \Delta x^{*}<0.25$. Hence, we consider that, in this range, the electromagnetic wave propagation can be simulated stably. However, for $y^{\text {shift }} \neq 0.0, r_{\mathrm{AD}}=1.0$ cannot be maintained in all cases even for $x^{\text {shift }}=0.0$. It must be noted here that, in all experiments, (40) is satisfied. Nevertheless, the unstable simulations have been observed. Hence, we consider that there are some type of constraints between the node alignment for $E$ and that for $H$, although we have not yet found the concrete constraints. To stably simulate electromagnetic wave propagation, not only the Courant condition but also the constraints may have to be satisfied.

\section{Conclusion}

To investigate a condition of the numerical stability of an electromagnetic wave propagation simulation by the MTDM, we have analyzed a 1D TM mode theoretically. Under some assumptions, governing equations of the 1D TM mode have been discretized with the explicit form of the derivatives of shape functions. By substituting the plane wave for the discretized equations, the equations can be rewritten by using a matrix and vectors. From the eigenvalues of the matrix, the Courant condition for the 1D MTDM has been derived theoretically. In addition, the Courant condition for the 2D/3D of the MTDM has been predicted as (40), based on the theoretical result for the $1 \mathrm{D}$ case. In numerical experiments, electromagnetic wave propagation in a line-shaped waveguide has been simulated by the 2D MTDM, for cases in which (40) is satisfied. Conclusions obtained in the present study are summarized as follows:

1. Under the assumptions described in Section 4, the Courant condition for the 1D MTDM was derived. In addition, the Courant condition does not depend on the RBFs and is equivalent to that for the FDTD.

2. Even though (40) is satisfied, the simulations may be unstable because of inappropriate node alignment.

In future study, the relation between the node alignment for $E$ and that for $H$ will be investigated to determine the concrete constraints for stable simulations. 


\section{Acknowledgments}

This work was partially supported by JSPS KAKENHI Grant Numbers 24700053 and 22360042. In addition, this work was supported in part by NIFS Collaboration Research Program (NIFS11KNTS011 and NIFS11KKGS009).
[1] T. Belytschko, Y.Y. Lu and L. Gu, Int. J. Numer. Meth. Engng. 37(2), 229 (1994).

[2] S.N. Atluri and T. Zhu, Comput. Mech. 22, 117 (1998).

[3] J.G. Wang and G.R. Liu, Int. J. Numer. Meth. Engng. 54, 1623 (2002).

[4] T. Kaufmann, C. Engström, C. Fumeaux and R. Vahldieck, IEEE Trans. Microw. Theory Tech. 58(12), 3399 (2010). 